\title{
Consensus statement on best practice management regarding the use of intravesical immunotherapy with BCG for bladder cancer
}

\author{
Ashish M. Kamat, Thomas W. Flaig, H. Barton Grossman, Badrinath Konety, Donald Lamm, \\ Michael A. O'Donnell, Edward Uchio, Jason A. Efstathiou and John A. Taylor III
}

\begin{abstract}
Multiple clinical trials have demonstrated that intravesical Bacillus Calmette-Guérin (BCG) treatment reduces recurrences and progression in patients with non-muscle-invasive bladder cancer (NMIBC). However, although BCG has been in use for almost 40 years, this agent is often underutilized and practice patterns of administration vary. This neglect is most likely caused by uncertainties about the optimal use of BCG, including unawareness of optimal treatment schedules and about patient populations that most benefit from BCG treatment. To address this deficit, a focus group of specialized urologic oncologists (urologists, medical oncologists and radiation oncologists) reviewed the current guidelines and clinical evidence, discussed their experiences and formed a consensus regarding the optimal use of BCG in the management of patients with NIMBC. The experts concluded that continuing therapy with 3-week BCG maintenance is superior to induction treatment only and is the single most important factor in improving outcomes in patients with NMIBC. They also concluded that a reliable alternative to radical cystectomy in truly BCG-refractory disease remains the subject of clinical trials. In addition, definitions for common terms of BCG failure, such as BCG-refractory and BCG-intolerant, have been formulated.
\end{abstract}

The University of Texas MD Anderson Cancer Center, 1515 Holcombe Boulevard, Unit 1373, Houston, TX 77030, USA (A.M.K., H.B.G.). University of Colorado, MS 8117 ,

12801 E. $17^{\text {th }}$ Avenue, Room 8117, Aurora, CO 80045, USA (T.W.F.) University of Minnesota, 420 Delaware Street Southeast, Minneapolis, MN 55455, USA (B.K.). BCG Oncology,

3815 East Bell Road Suite 1210, Phoenix, AZ 85032, USA (D.L.). University of lowa, 200 Hawkins Drive, lowa City, IA 52242

USA (M.A.O.). University of California Irvine, 333 City Boulevard, Suite 2100, Orange, CA 92868, USA (E.U.). Harvard Medical School, 100 Blossom Street, Cox 3, Boston, MA 02114, USA (J.A.E.). University of

Connecticut Health

Center, 263 Farmington Avenue, Farmington, CT 06030, USA (J.A.T.).

Correspondence to:

A.M.K.

akamat@

mdanderson.org

Kamat, A. M. et al. Nat. Rev. Urol. 12, 225-235 (2015); published online 24 March 2015; doi:10.1038/nrurol.2015.58

\section{Introduction}

Over the past decade, interest in the use of immunotherapy for cancer treatment has been steadily growing. Early results from clinical trials investigating immunomodulation with the programmed death ligand 1 (PD-L1) have contributed to this enthusiasm, particularly in the field of bladder cancer research. Experts will recognize, however, that immunotherapy for bladder cancer has been successfully used for almost 40 years. BCG was first introduced into clinical use in urology in 1976 and remains the most effective form of intravesical treatment for non-muscle-invasive bladder cancer (NMIBC). Despite this successful history, BCG therapy is internationally underutilized and practice patterns of BCG administration vary widely, probably because of misconceptions about its optimal use. ${ }^{1}$ This Expert Consensus Document provides a comprehensive review of immunomodulatory therapy with BCG, clarifies topics that can create confusion and recommends best practice guidelines to improve overall use and patient outcomes (Box 1).

\section{Methods}

Contributing authors were invited by the Leo \& Anne Albert Institute for Bladder Cancer Care and Research

Competing interests

The authors declare no competing interests. to partake in a symposium designed to evaluate and comment on current practices in bladder cancer. Participants were selected based on their expertise and knowledge in the area of interest. Global literature searches were performed for each section and presentations made with subsequent group discussion and consensus development based on the strength of the data.

\section{Historical perspective}

BCG is a live, attenuated form of the slow-growing, aerobic bovine tuberculosis bacterium, Mycobacterium bovis. In 1900, Albert Léon Charles Calmette and Camille Guérin of the Pasteur Institute in Lille, France, began work on a vaccine against Mycobacterium tuberculosis, one of the species that can cause tuberculosis in humans. They discovered that $M$. bovis is less virulent after being subcultured in a bile-containing medium. Now Middlebrook 7H9 medium is usually used. From 1908 to 1913 repeated subculturing of M. bovis resulted in a nonvirulent strain and vaccine trials in humans began in 1921. Before lyophilized storage in 1960, BCG was passaged continually (over 1,000 times), which led to the formation of multiple daughter strains-each strain a genetic variant of the original. Many of these mutants are used today, leading to different possible product characteristics and strengths, as well as varying dosing requirements. Remarkably, as demonstrated by 
Box 1 | Recommendations for intravesical BCG

- RCTs and practice pattern research demonstrate that BCG immunotherapy in NMIBC reduces recurrences and progression, and affects mortality

- 3-week BCG maintenance is confirmed to reduce recurrence rates compared with induction alone, as well as metastasis and mortality compared with maintenance chemotherapy; thus, it is the optimal regimen for current practice

- BCG maintenance schedules other than the 3-week schedule show no significant benefit in RCTs

- In the period of around 1.5-2 years after the identification of high-grade NMIBC nonradical alternative treatments for patients experiencing BCG-failure can be explored

- After the first BCG failure, patients (who have not progressed) have several treatment options, including repeated BCG (or continued maintenance), BCG plus interferon, single-agent intravesical chemotherapy (for example, mitomycin, gemcitabine, or valrubicin), sequential chemotherapy (for example, gemcitabine-docetaxel) or device-assisted chemotherapy

- After the second BCG failure, or if the disease is BCG-refractory, radical cystectomy should be considered with alternatives (listed above) considered a matter of investigation by clinical trials

- Patients with BCG-refractory disease who are not candidates for cystectomy can be considered for chemoradiation

Abbreviations: NMIBC, non-muscle-invasive bladder cancer; RCTs, randomized controlled trials. the high complete response rates observed with BCG preparations containing sufficient numbers of live bacteria, all strains are effective and clearly superior to standard intravesical chemotherapy. Although controlled clinical trials might confirm the superiority of one preparation over another, current shortages of BCG require that we assure both patients and government agencies that current experience suggests that all current BCG preparations are highly effective and superior to non-BCG alternatives.

The first report of BCG as an immunomodulator in cancer therapy was published in $1959 .{ }^{2}$ In 1976, Morales and colleagues ${ }^{3}$ published a landmark paper on the favourable effect of intravesical BCG on outcomes in recurrent superficial bladder cancer in nine patients. The first controlled trial showing similar results was published in 1980 and BCG received FDA approval for the treatment of superficial bladder cancer in $1990 .{ }^{4}$

\section{Review of current guidelines on BCG use}

Several guidelines exist regarding the use of BCG in NMIBC, such as those by the American Urological Association (AUA), ${ }^{5}$ the European Association of Urology (EAU), ${ }^{6}$ the National Comprehensive Cancer Network $(\mathrm{NCCN}),{ }^{7}$ the International Bladder Cancer Group $(\mathrm{IBCG})^{8}$ and the International Consultation on Urological Diseases (ICUD). ${ }^{9}$ Even though each of these guidelines is based on large-scale literature reviews on similar available data, the guidelines are somewhat disparate, making implementation of the recommendations into routine practice difficult for the practicing urologist.

\section{Low-grade tumours}

Consensus exists for small, solitary, superficial low-grade tumours $(\mathrm{Ta})$ with all groups agreeing that BCG is not indicated in this setting given the very low risk of disease progression. For multiple and/or large or recurrent low-grade tumours (the intermediate-risk category), BCG therapy with or without maintenance is considered optional by EAU and AUA guidelines and the IBCG $.5,6,8$ The AUA's meta-analysis suggests $24 \%$ and $31 \%$ reduction in recurrence rates for BCG induction and maintenance, respectively. ${ }^{5}$ The EAU recommendation is based predominantly on the recent meta-analysis by Sylvester and colleagues, ${ }^{10}$ which demonstrated a $32 \%$ relative risk reduction with the use of BCG maintenance compared with intravesical chemotherapy. The IBCG recommendation is based on a risk assessment model. ${ }^{11}$ By contrast, the NCCN guidelines do not specifically address these intermediate-risk tumours. ${ }^{7}$

\section{High-grade tumours (including carcinoma in situ)}

The recommendations for use of BCG in high-grade tumours are relatively consistent across guidelines; variations mainly exist in the recommended duration of therapy. The AUA, EUA and IBCG advise BCG induction with 1-3 years of maintenance for all high-grade tumours. ${ }^{5,6,8}$ ICUD guidelines for Ta high-grade tumours do not include maintenance BCG, remarking a lack of conclusive evidence about the effect of maintenance BCG on disease progression in these tumours. In addition, the value of maintenance therapy in T1 disease is questioned. ${ }^{9}$ Carcinoma in situ (CIS) is the only pathology for which maintenance is advised by ICUD and the guideline also includes reinduction of BCG instillations if no response is detected at first evaluation. ${ }^{9}$ The NCCN recommends BCG as standard treatment for CIS only, but lists BCG as the favoured option for high-grade Ta and T1 tumours and offers that maintenance should be considered.?

\section{Optimal schedule and duration of therapy}

The 6-week course of intravesical and percutaneous BCG, which was the first reported schedule, ${ }^{3}$ is very effective. Most subsequent studies have failed to demonstrate that alternative schedules provide significant benefits. Early modifications, such as omission of percutaneous vaccination, and quarterly or monthly maintenance, though accepted, only marginally improve convenience or efficacy. Although randomized clinical trials (RCTs) failed to demonstrate improved efficacy of percutaneous BCG against bladder cancer, systemic immune responses are higher after percutaneous vaccination than after intravesical administration alone. This heightened response after immunization is promising and should prompt new RCTs to investigate this application method.

RCTs of quarterly, monthly and repeated 6-week BCG instillations, which aim to maintain the immune response, also failed to show a significant improvement of efficacy (Figure 1). These results led some experts to question the value of maintenance BCG and to abandon its use. By contrast, the 3-week BCG regimen, designed by the Southwest Oncology Group with immunological principles in mind, ${ }^{12}$ is sufficiently different that it should not be considered in the same category as other maintenance $B C G$ regimens. 

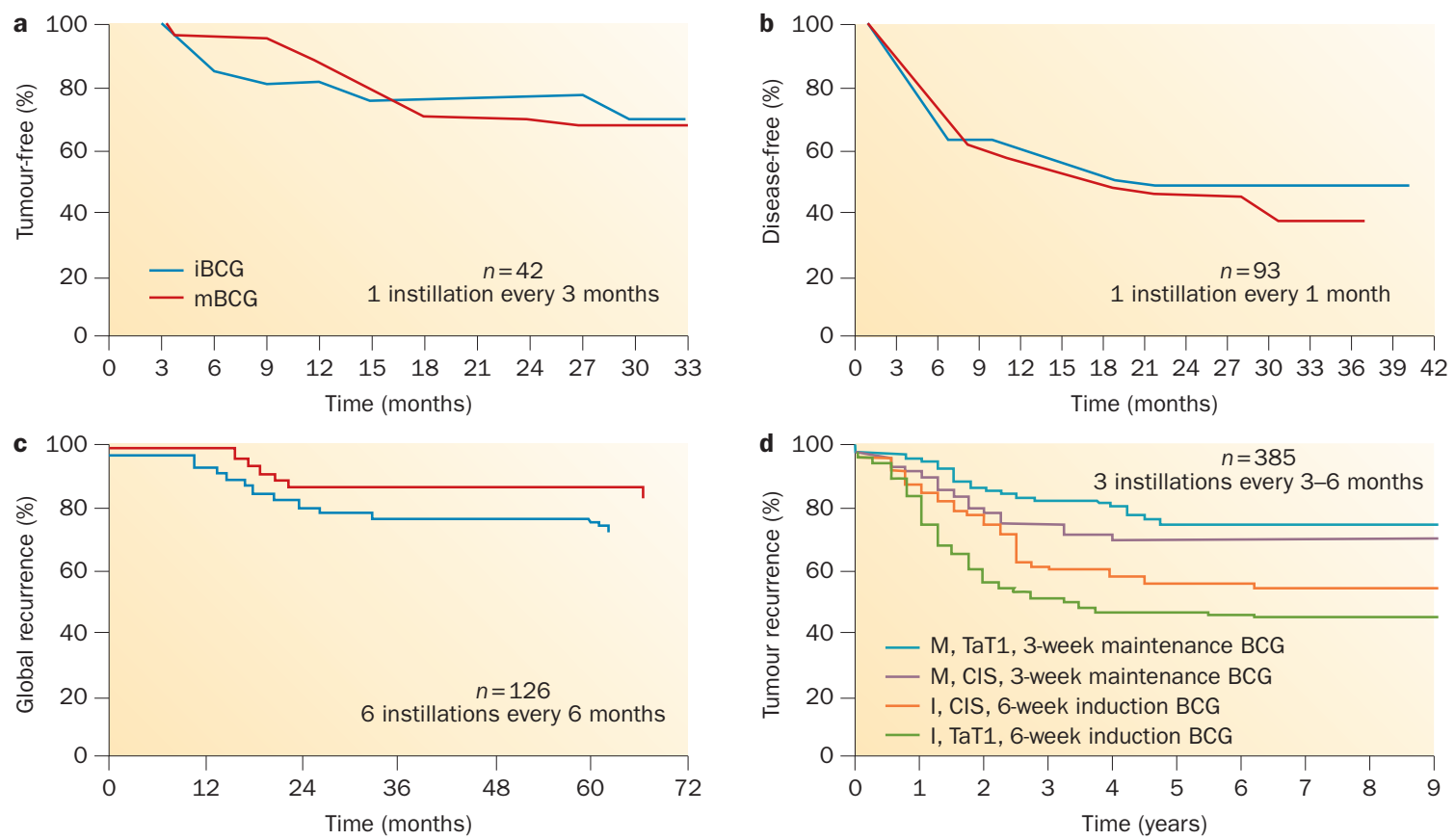

Figure 1 Not all maintenance schedules are equal. a,b,c | Kaplan-Meier curves demonstrate that recurrence trends in three clinical trials show no difference between BCG induction alone compared to BCG maintenance (suboptimal schedule). ${ }^{14,15,17} \mathbf{~ d ~ | ~ K a p l a n - M e i e r ~ c u r v e s ~ r e f l e c t ~ t h e ~ i m p o r t a n c e ~ o f ~ t r u e ~ m a i n t e n a n c e ~ t h e r a p y ~ o n ~ t u m o u r ~ r e c u r r e n c e ~ f o r ~}$ superficial bladder cancer of all stages. ${ }^{12}$ Abbreviations: CIS, carcinoma in situ; I, induction; M, maintenance. This figure is not covered by the CC-BY-NC-SA 4.0 licence. Reprinted from European Urology, 65, Kamat, A. M. \& Porten, S. Myths and mysteries surrounding bacillus Calmette-Guérin therapy for bladder cancer, 267-269, 2014, with permission from Elsevier.

The potential benefit of 3-week BCG maintenance deserves emphasis. Specifically, the schedule is initiated with weekly intravesical and percutaneous BCG treatment for 6 weeks, followed by further weekly combination treatment for 3 weeks after routine cystoscopy at 3, $6,12,18,24,30$ and 36 months. ${ }^{12}$ Only $14 \%$ of patients completed treatment in the original study, but compliance in modern studies is high. In the population with CIS, complete response at 6 months in patients randomized to receive the initial 6-week BCG treatment only (induction arm) was $69 \%$ in comparison with $84 \%$ $(P<0.01)$ in patients randomized to receive the additional 3-week maintenance treatment (maintenance arm). ${ }^{12}$ In the maintenance arm, complete response increased from $55 \%$ at 3 months to $84 \%$ at 6 months (64\% complete response in patients with treatment failure at 3 months). Even without additional BCG, $26 \%$ of patients with residual disease at 3 months in the induction arm went on to have complete response by 6 months, illustrating that 6 months is a preferred time to evaluate response.

Significant improvement in the treatment of CIS was confirmed by other RCTs with 3-week maintenance BCG, but no other treatment has level one evidence of superiority over induction BCG. The most commonly used treatment, repeated 6-week instillations, has been found to be ineffective in one RCT. ${ }^{13}$ Five RCTs compared non-3-week maintenance schedules to BCG induction only (Table 1). ${ }^{14-18}$ None of these alternative maintenance schedules showed a statistically significant reduction in recurrence (range $-4 \%$ to $22 \%$, average $7 \%$ ). By contrast, two studies that compared 3-week maintenance to induction treatment alone showed a statistically significant $28 \%$ reduction in tumour recurrence (Table 2). ${ }^{12,19}$ Moreover, only the 3 -week BCG maintenance schedule reduced disease progression and metastasis, as well as overall and cancer-specific mortality in RCTs. ${ }^{10,12}$

Two other trials compared 3-week maintenance BCG with alternative agents (Table 2). ${ }^{10,20}$ EORTC 30911 compared the 3-week maintenance BCG with 3-week maintenance epirubicin chemotherapy. BCG significantly reduced recurrence by $15 \%$, metastasis by $45 \%$ $(P=0.046)$, and both overall $(P=0.023)$ and cancerspecific mortality $(P=0.026) .{ }^{10}$ No other intravesical treatment, BCG induction only, or non-3-week maintenance BCG schedule has achieved such success.

The life-saving benefit of BCG immunotherapy in RCTs is confirmed in real-world practice pattern research. In a review of data from nearly 24,000 patients with bladder cancer from the Surveillance, Epidemiology, and End Results (SEER)-Medicare database, use of BCG significantly reduced mortality (HR 0.87), and bladder cancer deaths were reduced by $23 \%$ in patients with high-grade tumours. ${ }^{21}$ Education might now be the most important next step in improving care in bladder cancer. The review found that only $22 \%$ of eligible patients received BCG, and only a fraction of those received the critically important 3 -week maintenance schedule. ${ }^{21}$ 


\begin{tabular}{lllllll} 
Table 1 | Non-3-week maintenance BCG versus induction BCG only \\
\hline & $\begin{array}{l}\text { Patients } \\
(\boldsymbol{n})\end{array}$ & Schedule & $\begin{array}{l}\text { RFS without } \\
\text { maintenance (\%) }\end{array}$ & $\begin{array}{l}\text { RFS with } \\
\text { maintenance (\%) }\end{array}$ & $\begin{array}{l}\text { Difference* } \\
(\%)\end{array}$ & $P$ \\
\hline Badalament et al. $(1987)^{14}$ & 93 & Monthly & 45 & 45 & 0 & NS \\
\hline Hudson et al. $(1987)^{15}$ & 42 & Quarterly & 71 & 67 & -4 & NS \\
\hline Akaza et al. $(1995)^{16}$ & 107 & Monthly & 74 & 78 & +4 & NS \\
\hline Palou et al. $(2001)^{17}$ & 126 & Biannualy & 74 & 85 & +11 & NS \\
\hline Koga et al. $(2010)^{18}$ & 51 & Quarterly & 74 & 96 & +22 & NS \\
\hline
\end{tabular}

*7\% average increase in RFS. Abbreviations: NS, not significant; RFS, recurrence-free survival.

\section{Practical issues of BCG administration}

Some practical points need to be considered when using BCG for bladder cancer treatment. BCG manufacturers recommend evaluating the tuberculosis status of the patient with a PPD (purified protein derivative) tuberculosis skin test before initiation of therapy, with some practitioners obtaining chest radiographs in all patients..$^{22}$ This procedure has never proven necessary, probably owing to the exceedingly low incidence of tuberculosis in developed nations, where most bladder cancer patients do not meet the criteria for disease screening as recommended by the Centers of Disease Control and Prevention. ${ }^{23}$ In fact, patients with a positive PPD test without active disease should not be excluded from BCG therapy, as the presence of a systemic immune response might help augment the antitumour response as noted above. ${ }^{24}$ In addition, patients with a positive PPD result were shown to display adverse effect profiles during BCG treatment that are similar to patients with a negative test. ${ }^{25}$

In general, BCG use in patients with significant immunosuppression should be avoided, owing to the infectious risk of BCG. However, other patients with mild immune impairment (for example, due to steroid use for chronic obstructive pulmonary disease), well controlled HIV, or mature transplants, as well as healthy elderly patients, can be treated successfully with minimal adverse effects and good efficacy. ${ }^{26,27}$ In addition, medications such as statins or antiplatelet agents are not a contraindication for BCG therapy. Although these drugs have a potential negative effect on the immune response to BCG treatment, neither had an effect on the clinical efficacy of BCG therapy in multiple clinical studies. ${ }^{27-32}$ Other relative contraindications are prosthetic valves or orthopaedic hardware. However, a large phase II study with BCG combination therapy reported no infectious complications in the absence of prophylactic antibiotics, suggesting this population represents a low-risk setting. ${ }^{33}$ This recommendation is in concordance with the American Heart Association's guideline against antibiotic prophylaxis for genitourinary procedures such as urethral catheterization utilized in BCG administration. ${ }^{34}$

The technique used for the administration of BCG therapy must optimize the contact duration of the mycobacterium to the bladder urothelium to achieve maximum treatment effect. These measures include minimizing fluid intake by the patient before treatment and assuring complete bladder drainage via a lubricated, atraumatically placed catheter immediately before BCG instillation under gravity. Additional rolling and positional manoeuvers by the patient after instillation are unnecessary for complete contact with the urothelium and distribution of BCG. Local symptoms of bladder irritability are quite common with $60 \%$ of patients in clinical trials experiencing dysuria. ${ }^{22,35}$ Symptom management, such as use of single-dose, short-term quinolones $6 \mathrm{~h}$ after BCG instillation and/or use of anticholinergics, has been shown to decrease moderate to severe adverse events in some published reports, ${ }^{36,37}$ and enables the majority (90\%) of patients to tolerate full-dose BCG without discontinuation of therapy. ${ }^{38}$

\section{Definition of BCG failure}

Recognizing when BCG has failed is another area that can lead to confusion. Although the assumption seems logical that any tumour recurrence after therapy is a 'BCG failure', not all patients in this population have a similar prognosis. This situation has immense implications on options for alternative therapy, including the need to proceed to cystectomy. Definitions to clarify what constitutes BCG failure have been published. ${ }^{39}$ Herr \& Dalbagni ${ }^{40}$ and O'Donnell \& Boehle ${ }^{41}$ suggested definitions of the terms BCG relapse, BCG-refractory and BCG-intolerant:

- BCG relapse is considered a recurrence of tumour after a period of disease-free status. Most experts agree that the time point for evaluation should be at 3 months for papillary tumours and 6 months for CIS (except when disease progression was observed at 3 months). Relapse can be further stratified as early ( $<1$ year after treatment), intermediate ( $1-2$ years) or late ( $>2$ years), as the disease-free interval is a prognostic variable; early-relapsing patients are more likely to progress and late-relapsing patients can possibly derive some benefit from reinduction with BCG.42

- BCG-refractory is the persistence of disease after adequate induction and one maintenance course of BCG. Of note, this category includes any progression in stage or grade by 3 months if patients received induction BCG only.

- BCG-intolerant is defined as the inability to tolerate at least one full induction course of BCG. The tumour recurs largely because of inadequate therapy, which does not have the same negative prognostic implications as a true BCG failure. 
Table 2 | Randomized trials of 3-week maintenance BCG

\begin{tabular}{|c|c|c|c|c|c|c|}
\hline Study & $\begin{array}{l}\text { Patients } \\
(n)\end{array}$ & Agents & $\begin{array}{l}\text { RFS without } \\
\text { maintenance (\%) }\end{array}$ & $\begin{array}{l}\text { RFS with } \\
\text { maintenance (\%) }\end{array}$ & Difference (\%) & $P$ \\
\hline \multicolumn{7}{|l|}{ vs 6-week induction only } \\
\hline Lamm et al. $(2000)^{12}$ & 384 & BCG & 47 & 75 & +28 & $<0.01$ \\
\hline Hinotsu et al. $(2011)^{19}$ & 110 & BCG & 65 & 93 & +28 & $<0.02$ \\
\hline \multicolumn{7}{|l|}{ vs other 3-week agents } \\
\hline Sylvester et al. $(2010)^{10}$ & 789 & Epirubicin & 47 & 62 & +15 & $<0.01$ \\
\hline Duchek et al. $(2010)^{20}$ & 250 & Epirubicin + IFN- $\alpha 2 b$ & 50 & 65 & +15 & $<0.01$ \\
\hline
\end{tabular}

The above definitions highlight a major problem with the design of current clinical trials for bladder cancer using intravesical BCG therapy: lack of a standard definition of treatment failure and the complexity and ambiguity associated with related terminology. The situation becomes even more complex when the adequacy of BCG therapy is considered, as induction BCG alone is considered suboptimal therapy. Thus, when designing clinical trials for intravesical therapy, most clinicians recognize that to be included in a BGCfailure trial patients should receive at least five of six doses of induction BCG and at least two of three doses of maintenance BCG. This requirement brings about its own challenge: the lack of a good control group (other than cystectomy) for true BCG failures. If patients have recurrent high-grade tumours after BCG therapy as listed above, the salvage rates with intravesical therapy remain low. Thus, no clinical trial that randomizes BCG-refractory patients to alternative conservative measures compared to the much more invasive treatment of radical cystectomy has been proposed or even felt to be feasible to date.

Improving enrolment in clinical trials of new intravesical therapies will require changes in study design and approach. One such proposal is based on early identification of patients who experience 'cytogenetic failure' of BCG therapy. This suggestion is based on a prospective clinical trial, in which fluorescence in situ hybridization (FISH) assays that detect aneuploidy and gene mutation in urine samples (UroVysion, Abbott Molecular) were performed serially during BCG therapy. The data suggested that patients with a positive result in the FISH assay and a negative 3-month cystoscopy are at such high risk of recurrence and progression that their disease can be classified as a cytogenetic BCG failure, even though they are clinically disease-free. ${ }^{43}$ Thus, a proposed clinical trial design might include randomizing such patients to further BCG (as is standard of care) or alternative therapy that has previously demonstrated robust activity in other phase II trials. ${ }^{44}$

\section{Predictors of response to $B C G$}

Markers of response to intravesical BCG fall into three broad categories: determination of response (presence of cancer), use of surrogate endpoint biomarkers and prediction of response. BCG is most frequently used to treat high-grade disease and assessing the response of
CIS specifically is challenging because it is often difficult to detect. A commonly used design is to determine response by cystoscopy, cytology and possibly bladder biopsy at 3 months and 6 months following a BCG induction regimen. However, the 3 -month assessment is problematic because of the well documented conversion of positive cytology at 3 months to negative cytology at 6 months, particularly after maintenance BCG. ${ }^{12}$ Fluorescence cystoscopy can improve detection of both CIS and papillary bladder cancer, but fluorescence can also be induced by inflammation. ${ }^{45}$ It remains to be seen whether fluorescence cystoscopy can reliably improve the determination of BCG response.

Cytology is a subjective test with variable performance; ${ }^{46}$ molecular tests that are more objective might yield better and more consistent performance in detecting visually occult bladder cancer. Patients who have abnormal results in the UroVysion assay in a urine sample obtained immediately before the last of the 6-week induction instillations have increased risk for tumour recurrence and progression. ${ }^{43}$

Cytokine production has been explored as a source of potential surrogate endpoint biomarkers. Increased levels of IL- 8 within $6 \mathrm{~h}$ and increased levels of IL-18 within $12 \mathrm{~h}$ of the first BCG dose correlate with response to BCG treatment. ${ }^{47}$ Detection of IL-2 in the patient's urine during induction and the first maintenance treatment is associated with a favourable response to BCG. ${ }^{48}$ Similarly, patients receiving BCG whose peripheral blood mononuclear cells produce IL-2 in response to challenge with phytohaemagglutinin are likely to have a favourable response to BCG. ${ }^{49} \mathrm{~A}$ team from the MD Anderson Cancer Center has developed a nomogram based on the measurement of multiple cytokines that predicts response with an area under a receiver operating characteristics curve value of $0.85 .^{50}$

Response to immunotherapy with BCG depends on complex molecular interactions between the patient and their cancer. Genomic variations in oxidative stress pathway genes might affect response to BCG. ${ }^{51}$ Similarly, polymorphisms in immune response genes in combination with clinicopathological features can be used to distinguish patient groups with varying risk of relapsefree survival after BCG treatment. ${ }^{52}$ This approach is supported by evidence of a specific gene signature that is associated with response to BCG..$^{53}$ Molecular analysis has defined clinically relevant subtypes of bladder 
cancer. ${ }^{54}$ These tumours differ in their response to chemotherapy, but it is not yet clear if these molecular phenotypes significantly differ in their response to BCG. Increasing knowledge of the multiple factors that influence what is clinically recognized as a BCG response is likely to result in improved instruments to assess this response.

\section{Options after BCG failure}

BCG treatment eventually fails in up to $50 \%$ of patients and in about half of those within the first 6 months. ${ }^{55}$ In tumours that do not respond to BCG anymore, conventional intravesical chemotherapy agents, such as doxorubicin, thiotepa and mitomycin, have limited activity. In addition, valrubicin, the only FDA-approved drug for BCG-refractory CIS, is only effective in $<10 \%$ of patients at 2 years and none with coincident stage T1 disease. ${ }^{56}$ Thus, the traditional approach after BCG treatment failure has been to advocate early cystectomy. ${ }^{57}$ However, several lines of evidence suggest a reasonably safe window of opportunity, probably up to 1.5-2 years after onset of the index high-grade tumour, in which other conservative, nonradical therapies might be tried without undue risk. ${ }^{58-61}$

Repeated BCG induction is of limited value. About one-third of patients who do not respond to one course of BCG will eventually achieve a durable response. However, after further courses, the typical success rate of repeated BCG is $<10-20 \%{ }^{41,62,63}$ After recognizing that $B C G$ exerts its effects via cytokine stimulation, as well as response of type $1 \mathrm{~T}$ helper cells $\left(\mathrm{T}_{\mathrm{H}} 1\right)$ and $\mathrm{T}$ helper cell recruitment, the addition of cytokine therapy to BCG treatment has been tried. For example, IFN- $\alpha 2 b$ functions by enhancing protein TRAIL release, augmenting the $\mathrm{T}_{\mathrm{H}} 1$ response, increasing IFN- $\gamma$ release and reducing the expression of inflammation-inhibiting proteins such as IL-10. ${ }^{64,65}$ Although intravesical IFN- $\alpha$ provides a 2-year diseasefree rate of only $12 \%$ for BCG-refractory CIS, ${ }^{66}$ several phase I and phase II studies indicate a robust response of around $50 \%$ for the combination of BCG with IFN- $a 2 b$ in patients who have not responded to BCG alone. However, not all patients have the optimum salvage response that is observed in those who initially responded to BCG and developed recurrent tumours in a delayed fashion ( $>1$ years later). ${ }^{67-70}$ Long-term studies of up to 5 years show that, although early responses to the BCG-interferon treatment were excellent, $64 \%$ of patients had relapsed at 5 years with $20 \%$ having evidence of disease progression. ${ }^{71}$ The combination of reduced-dose BCG with IFN- $\alpha$ has shown favourable long-term results in first-time BCG failures. ${ }^{70,72}$ By contrast, the only randomized study of the combination of BCG with IFN- $a 2 b$ in BCG-naive patients failed to demonstrate any superiority of the combination over BCG alone, even though the tolerability of reduced dose BCG-IFN- $\alpha 2 b$ seemed to be better than that of full-dose BCG alone. ${ }^{73}$ Nevertheless, the lack of RCTs and lack of superiority of BCG-IFN versus BCG alone in BCG-naive patients has limited the widespread use of this combination treatment. Furthermore, even this therapy has unsatisfactory activity in BCG-refractory patients. The combination of BCG with IL-12, which is known to promote $\mathrm{T}_{\mathrm{H}} 1$ response and interferon release, has been studied in mice. Intravesical administration of a combination of BCG and IL-12 resulted in higher urine and serum levels of IFN- $\gamma$, especially after the third instillation, in comparison to either agent alone. ${ }^{74}$ Long-term outcome data for this treatment combination are not available.

Other alternative strategies have been developed for patients who experience a failure of BCG therapy. For example, the use of initial chemotherapy followed by BCG, the reverse version or alternating application, as well as other novel chemotherapy regimens, have been investigated in small clinical studies. ${ }^{55}$ In addition, several original approaches using electromotive acceleration have been investigated. Kaasinen et al. ${ }^{75}$ conducted a study in 236 patients with recurrent Ta or T1 bladder tumours. Eligible patients received five weekly instillations of mitomycin $\mathrm{C}$ and were subsequently randomized to monthly BCG only or alternating BCG and IFN- $\alpha 2 b$. They found a significantly lower recurrence rate in the arm treated with mitomycin $C$ followed by BCG only, indicating no benefit of IFN- $\alpha 2 b$ in this situation. The use of hyperthermic conditions with intravesical mitomycin $\mathrm{C}$ can increase the response rate compared with euthermic conditions. Hyperthermic conditions resulted in a significant improvement in long-term disease-free survival. ${ }^{76}$ In another combination approach, Di Stasi et al. ${ }^{77}$ administered electromotive mitomycin $\mathrm{C}$ alternating with BCG compared with BCG alone in a complicated schedule that also included maintenance therapy in the complete responders. In this randomized trial, patients receiving the combination therapy demonstrated a significantly lower recurrence rate $(42 \%$ versus $58 \%, P=0.0012$ ). The randomized EORTC-30993 trial compared sequential mitomycin $\mathrm{C}$ and BCG to BCG alone, but did not demonstrate any benefit of the combination treatment in terms of recurrence or progression. ${ }^{78}$ Results from an aggregate meta-analysis did not show any difference in response rates or progression between administering chemotherapy and BCG concomitantly or in sequence. ${ }^{79}$ However, the combination approach did trend towards reduced recurrence (relative risk 0.75) and progression (relative risk 0.45 ) for patients with $\mathrm{Ta}$ or T1 tumours, while not having much benefit in those with CIS.

Several approved chemotherapeutics that are active in advanced disease, including cisplatin, gemcitabine and taxanes, are being studied for intravesical use. Unfortunately, the most active single systemic agent, cisplatin, is associated with unpredictable and sometimes fatal anaphylaxis when used intravesically. ${ }^{80}$ By contrast, intravesical gemcitabine is particularly active in patients with intermediate risk papillary disease in whom BCG treatment has failed, even demonstrating superiority in comparison with mitomycin. ${ }^{81,82}$ However, two studies of gemcitabine in BCG-refractory disease resulted in 2 -year durable responses of only $7 \%$ and $21 \% .^{83,84}$ 
Intravesical docetaxel has shown good activity, even in BCG-refractory NMIBC. Among 54 patients, 59\% experienced a complete response, $40 \%$ were diseasefree at 12 months, and $25 \%$ at 3 years. Cystectomy was required in $24 \%$ with an overall 5 -year disease-specific survival of $85 \% .{ }^{85}$

Intravesical multi-agent chemotherapy in NMIBC has only recently been investigated. Past combinations of harsher, vesicant drugs, such as doxorubicin and mitomycin, although highly active, resulted in considerable local chemical cystitis. ${ }^{86}$ By contrast, newer nonvesicant drugs, such as gemcitabine and docetaxel, are very well tolerated. ${ }^{87,88}$ Sequential gemcitabine-mitomycin C in 47 patients with high-risk disease, primarily after BCG failure, resulted in a complete response in $68 \%$ of patients with 1-year and 2-year disease-free survival of $48 \%$ and $38 \%$, respectively. ${ }^{89}$ Alternatively, sequenced gemcitabine-docetaxel in 45 patients with high-risk disease yielded a complete response rate of $66 \%$, as well as 1-year and 2-year recurrence-free survival of $54 \%$ and $34 \%$, respectively. ${ }^{90}$ Reassuringly, although the cystectomy rate for both regimens was around $20 \%$, metastasis was rarely reported.

\section{Radiation as an option for recurrent disease}

Cystectomy remains the standard for recurrent BCGrefractory, high-grade T1 bladder tumours. However, many patients (especially the elderly) are not undergoing surgery, possibly owing to comorbidities and/ or a desire to avoid a large operation. ${ }^{91}$ Furthermore, the high rates of clinicopathological stage discordance noted after surgery (around $45 \%$ of $\mathrm{T} 1$ tumours are being upstaged) might contribute to the poor longterm results of second-line intravesical agents following BCG failure. ${ }^{92}$

Radiation therapy presents a non-surgical option for BCG failures. Early studies showed reasonable response rates (48-69\%), ${ }^{93,94}$ and one randomized trial in patients with high-grade $\mathrm{T} 1$ tumours comparing conservative treatment with radiation alone showed no difference between treatments in terms of recurrence. ${ }^{95}$

There is increasing data on combined radiochemotherapy for high-risk NMIBC following maximum transurethral resection of the bladder tumour (TURBT). One study compared radiotherapy or radiochemotherapy in 141 patients and reported a complete response rate of $88 \%$; at 5 years and 10 years, overall progression rates were $19 \%$ and $30 \%$ (13\% and $29 \%$ for high-grade T1 tumours) and overall disease-specific survival was $82 \%$ and $73 \%$ (89\% and $79 \%$ for complete responders, $80 \%$ and $71 \%$ for high-grade T1 tumours), respectively. ${ }^{96}$ Over $80 \%$ of survivors could retain their native bladder and around $70 \%$ were "delighted" or "pleased" with their urinary function. A second study reported on 17 patients undergoing trimodality therapy (TURBT, radiation and chemotherapy) following T2 recurrence after failure of BCG for non-invasive disease. ${ }^{97}$ With 7 years follow-up time, only one patient required cystectomy, 10 patients (59\%) were free of any bladder tumour recurrence and disease-specific survival was $70 \%$.
Based on current evidence, the new 2015 NCCN bladder cancer guideline states that external-beam radiotherapy is rarely appropriate for patients with stage Ta, T1, or Tis disease. Radiochemotherapy might be a potentially curative alternative to cystectomy in patients with recurrent Ta-T1 disease (usually following BCG therapy but without extensive Tis). ${ }^{98}$ Currently, the North American multicentre, cooperative Radiation Therapy Oncology Group (RTOG) protocol RTOG 0926 is evaluating the role of radiochemotherapy $(61.2 \mathrm{~Gy}$ with concurrent cisplatin or 5-fluorouracil plus mitomycin C) after maximum TURBT for patients with high-risk T1 bladder cancer following BCG failure for whom the next therapy would have been radical cystectomy. ${ }^{99}$

\section{Novel agents}

Currently, several strategies to enhance BCG response are being explored. Testing of combinations of BCG with various new investigational agents that have independently demonstrated activity in early phase trials is ongoing and some of these combinations have been applied clinically with variable effect. In addition, the use of entirely novel agents based on emerging preclinical findings presents an important area for potential clinical investigation.

Some bladder cancer cells have a high level of VEGF expression. Tyrosine kinase inhibitors, such as sunitinib, can inhibit proliferation of such cells. In vitro experiments using T24 bladder cancer cells have demonstrated that the combination of sunitinib and BCG can enhance cell death and reduce cell migration compared with sequential administration. ${ }^{100}$ It remains to be seen whether similar responses can be obtained in vivo or in humans. Smac mimetics degrade inhibitors of apoptosis (IAP) proteins, which can block caspase-induced apoptotic death, and have been studied for augmenting response to BCG. ${ }^{101}$ One of the mechanisms of action of BCG is to enhance release of protein TRAIL from neutrophils, which can be inhibited by IAP proteins. ${ }^{102,103}$ In one in vitro study, application of BCG-conditioned neutrophil medium along with a small molecule Smac mimetic resulted in enhanced cytotoxicity compared with the use of BCG alone. ${ }^{104}$ Finally, trapping of BCG by exogenously introduced fibrinogen can enhance local concentration of BCG and uptake via fibronectin. This strategy has been shown to enhance BCG response in vitro. ${ }^{105}$

Other developments in oncology might also affect the future of intravesical treatment in bladder cancer. The immune checkpoint inhibitors ipilimumab and pembrolizumab (antibodies that target CTLA-4 and PD-1, respectively) have been approved for use in advanced melanoma. The receptor PD-1 is one checkpoint of T-cell function and is activated by PD-L1 and PD-L2. Anti-CTLA-4 and anti-PD-1 antibodies can prolong and enhance the T-cell response. In bladder tumours, PD-L1 expression has been correlated with bladder cancer T-stage and is highest in T3/T4 and CIS. ${ }^{106}$ In addition, immunohistochemical data suggest that PD-L1 expression is increased in tumours from patients with 
BCG failure. ${ }^{106}$ PD-L1 and PD-L2 can also be inhibited and could provide an alternative strategy, as some evidence exists that inhibition of PD-L1 might avoid autoimmune responses, which are usually modulated by PD-L2. IDO-1 inhibitors can work in a fashion similar to PD-1 inhibitors to enhance $\mathrm{T}_{\mathrm{H}}$ activity. One such inhibitor, indoximod is currently being studied in a clinical trial for advanced prostate cancer and could potentially be used together with BCG to enhance response. ${ }^{107}$ Currently, immune checkpoint inhibitors for the treatment of bladder cancer are being evaluated in the advanced-disease setting, but they might also have a role in early-stage disease in combination with BCG.

Intravesical therapy with a conditionally replicating adenovirus that was genetically engineered to express GM-CSF was well tolerated and induced a local immune response. ${ }^{108}$ Instillation of a nonreplicating adenoviral vector encoding IFN- $\alpha 2 b$ together with the exipient Syn3 to enhance gene transfer also seemed to be well tolerated and could yield responses. ${ }^{109}$ Photodynamic therapy is another novel approach to bladder cancer therapy. Several early studies suggested a benefit and one small study in 34 patients reported a favourable disease-free recurrence rate. ${ }^{110-112}$ However, despite the novelty in these and other approaches, none have been evaluated in a definitive, practice-changing study or are currently considered standard therapy.

Another treatment for NMIBC are toxic protein conjugates. These agents contain both a targeting and a treatment component to increase specificity for cancer cells. One clinical example of this approach is denileukin diftitox, a fusion of the diphtheria toxin and IL-2, which targets a membrane receptor that is differentially expressed in target cancer cells compared with the normal cells. ${ }^{113}$ In bladder cancer, oportuzumab monatox, a recombinant fusion protein of an antiEp-CAM antibody with the pseudomonas exotoxin, has been evaluated. In a phase II study in 46 BCG-refractory patients with CIS, $44 \%$ achieved a complete response with this therapy. ${ }^{114}$ Other preclinical evaluations of this approach include targeting diphtheria toxin to the epidermal growth factor receptor (EGFR), which is frequently overexpressed in bladder cancer but not the normal urothelium. ${ }^{115}$ Diphtheria toxin linked to EGF has shown encouraging results in murine orthotopic models of bladder cancer without any clear systemic or urothelial toxicity. ${ }^{116}$

Nanotechnology can be directed to specific cells and then heated for focal thermal ablation with the application of external light energy. In bladder cancer therapy, direct intravesical delivery can avoid problems caused by systemic clearance. Gold nanorods conjugated to anti-EGFR antibodies have been tested in bladder cancer cell lines using near-infrared light. ${ }^{117} \mathrm{~A}$ similar approach has been developed using gold nanorods targeting the fibroblast growth factor receptor, highlighting the generalizability of this approach based on the expression of target molecules on tumour cells. ${ }^{118}$

One approach to address the toxicity observed with BCG treatment is the use of nonlive-BCG immunotherapy. For example, BCG cell wall components have been incorporated into octaarginine-modified liposomes, which have similarities to an envelope-type virus, for cellular delivery. ${ }^{119}$ In fact, a similar strategy was used in the MCNA agent, in which mycobacterial cell wall is complexed to nuclear DNA with the aim of achieving both immunological and direct cytotoxic effects. A recent study in 129 patients with high-risk disease after BCG failure suggested a high activity of this agent. ${ }^{120}$ Overall disease-free rate was $25.0 \%$ at 1 year and $19.0 \%$ at 2 years. In patients with papillary-only tumours, MCNA was even more effective with rates of $35.1 \%$ and $32.2 \%$ at 1 year and 2 years, respectively.

\section{Conclusion}

Immunotherapy for patients with NMIBC remains the gold standard, with efficacy of intravesical BCG surpassing that of chemotherapy. In order for BCG immunotherapy to be effective, however, practitioners must learn (or re-learn) how to use it in an optimal manner. First, patient selection is paramount and complete resection of all visible tumours is important, not only for staging, but also to improve outcomes. BCG induction with 6-week instillations must be followed by 3-week maintenance instillations, as recommended in the SWOG protocol, as no other maintenance schedule has been shown to work reliably. If a patient develops recurrence on BCG therapy, the timing and tumour characteristics of this recurrence should be considered when classifying the patient in the subgroups within the BCG-failure category. Although radical cystectomy remains the mainstay for patients with high-risk BCG failure, for those who are unfit or who refuse cystectomy, several alternative options exist as outlined in this Expert Consensus Document. Future strategies aimed at improving the immune response of patients (with or without BCG), device-assisted chemotherapy delivery and combination chemotherapy all seem to be promising alternatives.
1. Witjes, J. A. et al. Current clinical practice gaps in the treatment of intermediate- and high-risk non-muscle-invasive bladder cancer (NMIBC) with emphasis on the use of bacillus Calmette-Guérin (BCG): results of an international individual patient data survey (IPDS). BJU Int. 112, 742-750 (2013).

2. Old, L. J., Clarke, D. A. \& Benacerraf, B. Effect of Bacillus Calmette-Guérin infection on transplanted tumours in the mouse. Nature 184 (Suppl. 5), 291-292 (1959).
3. Morales, A., Eidinger, D. \& Bruce, A. W. Intracavitary Bacillus Calmette-Guérin in the treatment of superficial bladder tumors. J. Urol. 116, 180-183 (1976).

4. Lamm, D. L. et al. Bacillus Calmette-Guérin immunotherapy of superficial bladder cancer. J. Urol. 124, 38-40 (1980).

5. Hall, M. C. et al. Guideline for the management of nonmuscle invasive bladder cancer (stages Ta, T1, and Tis): 2007 update. J. Urol. 178, 2314-2330 (2007).
6. Babjuk, M. et al. EAU guidelines on non-muscle-invasive urothelial carcinoma of the bladder: update 2013. Eur. Urol. 64, 639-653 (2013).

7. Clark, P. E. et al. Bladder cancer. J. Natl Compr. Canc. Netw. 11, 446-475 (2013).

8. Lamm, D. et al. Clinical practice recommendations for the management of nonmuscle invasive bladder cancer. Eur. Urol. Suppl. 7, 651-666 (2008).

9. Burger, M. et al. ICUD-EAU International Consultation on Bladder Cancer 2012: 
non-muscle-invasive urothelial carcinoma of the bladder. Eur. Urol. 63, 36-44 (2013).

10. Sylvester, R. J. et al. Long-term efficacy results of EORTC genito-urinary group randomized phase 3 study 30911 comparing intravesical instillations of epirubicin, bacillus Calmette-Guérin, and bacillus Calmette-Guérin plus isoniazid in patients with intermediate- and high-risk stage Ta T1 urothelial carcinoma of the bladder. Eur. Urol. 57, 766-773 (2010).

11. Kamat, A. M. et al. Defining and treating the spectrum of intermediate risk nonmuscle invasive bladder cancer. J. Urol. 192, 305-315 (2014).

12. Lamm, D. L. et al. Maintenance bacillus Calmette-Guérin immunotherapy for recurrent TA T1 and carcinoma in situ transitional cell carcinoma of the bladder: a randomized Southwest Oncology Group Study. J. Urol. 163, 1124-1129 (2000).

13. Gandhi, N. M., Morales, A. \& Lamm, D. L. Bacillus Calmette-Guérin immunotherapy for genitourinary cancer. BJU Int. 112, 288-297 (2013)

14. Badalament, R. A. et al. A prospective randomized trial of maintenance versus nonmaintenance intravesical bacillus CalmetteGuérin therapy of superficial bladder cancer. J. Clin. Oncol. 5, 441-449 (1987).

15. Hudson, M. A. et al. Single course versus maintenance bacillus Calmette-Guérin therapy for superficial bladder tumors: a prospective, randomized trial. J. Urol. 138, 295-298 (1987).

16. Akaza, H., Hinotsu, S., Aso, Y., Kakizoe, T. \& Koiso, K. Bacillus Calmette-Guérin treatment of existing papillary bladder cancer and carcinoma in situ of the bladder. Four-year results. The Bladder Cancer BCG Study Group. Cancer 75, 552-559 (1995)

17. Palou, J. et al. Control group and maintenance treatment with bacillus Calmette-Guérin for carcinoma in situ and/or high grade bladder tumors. J. Urol. 165, 1488-1491 (2001).

18. Koga, H. et al. Maintenance intravesical bacillus Calmette-Guérin instillation for Ta, T1 cancer and carcinoma in situ of the bladder: randomized controlled trial by the BCG Tokyo Strain Study Group. Int. J. Urol. 17, 759-766 (2010).

19. Hinotsu, S. et al. Maintenance therapy with bacillus Calmette-Guérin Connaught strain clearly prolongs recurrence-free survival following transurethral resection of bladder tumour for non-muscle-invasive bladder cancer. BJU Int. 108, 187-195 (2011).

20. Duchek, M. et al. Bacillus Calmette-Guérin is superior to a combination of epirubicin and interferon- $\alpha 2 b$ in the intravesical treatment of patients with stage T1 urinary bladder cancer. A prospective, randomized, Nordic study. Eur. Urol. 57, 25-31 (2010)

21. Spencer, B. A. et al. Adjuvant intravesical bacillus Calmette-Guérin therapy and survival among elderly patients with non-muscle-invasive bladder cancer. J. Oncol. Pract. 9, 92-98 (2013).

22. Merck Sharp \& Dohme B.V. TICE ${ }^{\circledR}$ BCG LIVE (for intravesical use) - Prescribing Information [online], http://www.merck.com/product/usa/ pi circulars/t/tice bcg/ticebcg pi.pdf (2013).

23. Bloch, A. B. Screeening for tuberculosis and tuberculosis infection in high-risk populations. Recommendations of the Advisory Council for the Elimination of Tuberculosis. MMWR Recomm. Rep. 44, 18-34 (1995).

24. Biot, C. et al. Preexisting BCG-specific T cells improve intravesical immunotherapy for bladder cancer. Sci. Transl. Med. 4, 137ra72 (2012).

25. Bilen, C. Y., Inci, K., Erkan, I. \& Ozen, H. The predictive value of purified protein derivative results on complications and prognosis in patients with bladder cancer treated with bacillus Calmette-Guérin. J. Urol. 169 1702-1705 (2003).

26. Gaughan, E. M. et al. HIV-associated bladder cancer: a case series evaluating difficulties in diagnosis and management. BMC Urol. 9, 10 (2009).

27. Joudi, F. N., Smith, B. J., O'Donnell, M. A. \& Konety, B. R. The impact of age on the response of patients with superficial bladder cancer to intravesical immunotherapy. J. Urol. 175, 1634-1639; discussion 1639-1640 (2006).

28. Hoffmann, P., Roumeguère, T., Schulman, C. \& van Velthoven, R. Use of statins and outcome of BCG treatment for bladder cancer. N. Engl. J. Med. 355, 2705-2707 (2006).

29. Kamat, A. M. \& Wu, X. Statins and the effect of BCG on bladder cancer. N. Engl. J. Med. 356, 1276; author reply 1276-1277 (2007).

30. Berglund, R. K., Savage, C. J., Vora, K. C., Kurta, J. M. \& Cronin, A. M. An analysis of the effect of statin use on the efficacy of bacillus Calmette-Guérin treatment for transitional cell carcinoma of the bladder. J. Urol. 180, 1297-1300; discussion 1300 (2008).

31. Crivelli, J. J. et al. Effect of statin use on outcomes of non-muscle-invasive bladder cancer. BJU Int. 112, E4-E12 (2013).

32. Gee, J. R. et al. Reduced bladder cancer recurrence rate with cardioprotective aspirin after intravesical bacille Calmette-Guérin. BJU Int. 103, 736-739 (2009).

33. Rosevear, H. M., Lightfoot, A. J., Nepple, K. G. \& O'Donnell, M. A. Safety and efficacy of intravesical bacillus Calmette-Guérin plus interferon $a-2 b$ therapy for nonmuscle invasive bladder cancer in patients with prosthetic devices. J. Urol. 184, 1920-1924 (2010).

34. Wilson, W. et al. Prevention of infective endocarditis: guidelines from the American Heart Association: a guideline from the American Heart Association Rheumatic Fever, Endocarditis, and Kawasaki Disease Committee, Council on Cardiovascular Disease in the Young, and the Council on Clinical Cardiology, Council on Cardiovascular Surgery and Anesthesia, and the Quality of Care and Outcomes Research Interdisciplinary Working Group. Circulation 116, 1736-1754 (2007).

35. Lamm, D. L. et al. Randomized intergroup comparison of bacillus Calmette-Guérin immunotherapy and mitomycin $\mathrm{C}$ chemotherapy prophylaxis in superficial transitional cell carcinoma of the bladder a Southwest Oncology Group study. Urol. Oncol. 1, 119-126 (1995).

36. Colombel, M. et al. The effect of ofloxacin on bacillus Calmette-Guérin induced toxicity in patients with superficial bladder cancer: results of a randomized, prospective, double-blind, placebo controlled, multicenter study. J. Urol. 176, 935-939 (2006).

37. Damiano, R. et al. Short-term administration of prulifloxacin in patients with nonmuscle-invasive bladder cancer: an effective option for the prevention of bacillus Calmette-Guérin-induced toxicity? BJU Int. 104, 633-639 (2009).

38. Oddens, J. et al. Final results of an EORTC-GU cancers group randomized study of maintenance bacillus Calmette-Guérin in intermediate- and high-risk Ta, T1 papillary carcinoma of the urinary bladder: one-third dose versus full dose and 1 year versus 3 years of maintenance. Eur. Urol. 63, 462-472 (2013).

39. Martin, F. M. \& Kamat, A. M. Definition and management of patients with bladder cancer who fail BCG therapy. Expert Rev. Anticancer Ther. 9, 815-820 (2009).
40. Herr, H. W. \& Dalbagni, G. Defining bacillus Calmette-Guérin refractory superficial bladder tumors. J. Urol. 169, 1706-1708 (2003).

41. O'Donnell, M. A. \& Boehle, A. Treatment options for BCG failures. World J. Urol. 24, 481-487 (2006).

42. Merz, V. W. et al. Analysis of early failures after intravesical instillation therapy with bacille Calmette-Guérin for carcinoma in situ of the bladder. Br. J. Urol. 75, 180-184 (1995).

43. Kamat, A. M. et al. Use of fluorescence in situ hybridization to predict response to bacillus Calmette-Guérin therapy for bladder cancer: results of a prospective trial. J. Urol. 187, 862-867 (2012).

44. Willis, D. L. \& Kamat, A. M. Clinical trials examining BCG treatment failure: improving trial design, options in intravesical therapy. 2013 Genitourinary Cancers Symposium Daily News [online], http://gucasym.org/clinical-trialsexamining-bcg-treatment-failure-improving-trialdesign-options-intravesical-therapy (2013).

45. Fradet, Y. et al. A comparison of hexaminolevulinate fluorescence cystoscopy and white light cystoscopy for the detection of carcinoma in situ in patients with bladder cancer: a phase III, multicenter study. J. Urol. 178, 68-73; discussion 73 (2007)

46. Glatz, K. et al. An international telecytologic quiz on urinary cytology reveals educational deficits and absence of a commonly used classification system. Am. J. Clin. Pathol. 126, 294-301 (2006).

47. Thalmann, G. N. et al. Urinary interleukin-8 and 18 predict the response of superficial bladder cancer to intravesical therapy with bacillus Calmette-Guérin. J. Urol. 164, 2129-2133 (2000).

48. Saint, F. et al. Urinary IL-2 assay for monitoring intravesical bacillus Calmette-Guérin response of superficial bladder cancer during induction course and maintenance therapy. Int. J. Cancer 107, 434-440 (2003).

49. Kaempfer, R. et al. Prediction of response to treatment in superficial bladder carcinoma through pattern of interleukin-2 gene expression. J. Clin. Oncol. 14, 1778-1786 (1996)

50. Kamat, A. M., Briggman, J., Dinney, C. P. $\&$ Urbauer, D. The use of a panel of urinary cytokines to predict response to BCG therapy for bladder cancer. WO/2013/131093 (2013).

51. Wei, H. et al. Association of polymorphisms in oxidative stress genes with clinical outcomes for bladder cancer treated with Bacillus Calmette-Guérin. PLoS ONE 7, e38533 (2012).

52. Lima, L. et al. The role of functional polymorphisms in immune response genes as biomarkers of BCG immunotherapy outcome in bladder cancer: establishment of a predictive profile in a Southern Europe population. BJU Int. http://dx.doi.org/10.1111/bju.12844.

53. Kim, Y. J. et al. Gene signatures for the prediction of response to Bacillus Calmette-Guérin immunotherapy in primary pT1 bladder cancers. Clin. Cancer Res. 16, 2131-2137 (2010).

54. Choi, W. et al. Identification of distinct basal and luminal subtypes of muscle-invasive bladde cancer with different sensitivities to frontline chemotherapy. Cancer Cell 25, 152-165 (2014).

55. Lightfoot, A. J., Rosevear, H. M. \& O'Donnell, M. A. Recognition and treatment of BCG failure in bladder cancer. ScientificWorldJournal 11, 602-613 (2011).

56. Steinberg, G. et al. Efficacy and safety of valrubicin for the treatment of Bacillus CalmetteGuérin refractory carcinoma in situ of the bladder. The Valrubicin Study Group. J. Urol. 163 761-767 (2000) 
57. Lebret, T. \& Neuzillet, Y. Indication and timing of cystectomy in high-risk bladder cancer. Curr. Opin. Urol. 22, 427-431 (2012).

58. Nepple, K. G. \& O'Donnell, M. A. The optimal management of T1 high-grade bladder cancer. Can. Urol. Assoc. J. 3 (Suppl. 4), S188-S192 (2009).

59. Cheng, L. et al. Survival of patients with carcinoma in situ of the urinary bladder. Cancer 85, 2469-2474 (1999).

60. Millán-Rodríguez, F. et al. Primary superficial bladder cancer risk groups according to progression, mortality and recurrence. J. Urol. 164, 680-684 (2000).

61. Herr, H. W. \& Sogani, P. C. Does early cystectomy improve the survival of patients with high risk superficial bladder tumors? J. Urol. 166 1296-1299 (2001)

62. Catalona, W. J., Hudson, M. A., Gillen, D. P., Andriole, G. L. \& Ratliff, T. L. Risks and benefits of repeated courses of intravesical bacillus Calmette-Guérin therapy for superficial bladder cancer. J. Urol. 137, 220-224 (1987).

63. Gacci, M. et al. Intravesical gemcitabine in BCGrefractory T1G3 transitional cell carcinoma of the bladder: a pilot study. Urol. Int. 76, 106-111 (2006).

64. Luo, Y., Chen, X., Downs, T. M., DeWolf, W. C. \& O’Donnell, M. A. IFN- $\alpha$ 2B enhances Th1 cytokine responses in bladder cancer patients receiving Mycobacterium bovis bacillus Calmette-Guérin immunotherapy. J. Immunol. 162, 2399-2405 (1999).

65. Fanger, N. A., Maliszewski, C. R., Schooley, K. \& Griffith, T. S. Human dendritic cells mediate cellular apoptosis via tumor necrosis factorrelated apoptosis-inducing ligand (TRAIL). J. Exp. Med. 190, 1155-1164 (1999).

66. Belldegrun, A. S. et al. Superficial bladder cancer: the role of interferon-a. J. Urol. 159, 1793-1801 (1998)

67. O’Donnell, M. A., Lilli, K., Leopold, C. \& National Bacillus Calmette-Guérin/Interferon Phase 2 Investigator Group. Interim results from a national multicenter phase II trial of combination bacillus Calmette-Guérin plus interferon $a-2 b$ for superficial bladder cancer. J. Urol. 172, 888-893 (2004).

68. Luciani, L. G., Neulander, E., Murphy, W. M. \& Wajsman, Z. Risk of continued intravesica therapy and delayed cystectomy in BCGrefractory superficial bladder cancer: an investigational approach. Urology 58, 376-379 (2001).

69. Lam, J. S. et al. Bacillus Calmete-Guérin plus interferon- $\alpha 2 \mathrm{~B}$ intravesical therapy maintains an extended treatment plan for superficial bladder cancer with minimal toxicity. Urol. Oncol. 21, 354-360 (2003).

70. Joudi, F. N., Smith, B. J., O’Donnell, M. A. \& National BCG-Interferon Phase 2 Investigator Group. Final results from a national multicenter phase II trial of combination bacillus CalmetteGuérin plus interferon $\alpha-2 \mathrm{~B}$ for reducing recurrence of superficial bladder cancer. Urol. Oncol. 24, 344-348 (2006).

71. Mohanty, N. K., Malhotra, V., Nayak, R. L. \& Arora, R. P. Combined low-dose intravesical immunotherapy (BCG + interferon $a-2 b)$ in the management of superficial transitional cell carcinoma of the urinary bladder: a five-year follow-up. J. Chemother. 14, 194-197 (2002).

72. Lamm, D., Brausi, M., O’Donnell, M. A. \& Witjes, J. A. Interferon $\alpha$ in the treatment paradigm for non-muscle-invasive bladder cancer. Urol. Oncol. 32, 35.e21-35.e30 (2014).

73. Nepple, K. G. et al. Bacillus Calmette-Guérin with or without interferon $a-2 b$ and megadose versus recommended daily allowance vitamins during induction and maintenance intravesical treatment of nonmuscle invasive bladder cancer. J. Urol. 184, 1915-1919 (2010)

74. O'Donnell, M. A. et al. Role of IL-12 in the induction and potentiation of IFN- $\gamma$ in response to bacillus Calmette-Guérin. J. Immunol. 163 4246-4252 (1999).

75. Kaasinen, E. et al. Weekly mitomycin C followed by monthly bacillus Calmette-Guérin or alternating monthly interferon- $\alpha 2 \mathrm{~B}$ and bacillus Calmette-Guérin for prophylaxis of recurrent papillary superficial bladder carcinoma. J. Urol. 164, 47-52 (2000).

76. Colombo, R., Salonia, A., Leib, Z., Pavone-Macaluso, M. \& Engelstein, D. Long-term outcomes of a randomized controlled tria comparing thermochemotherapy with mitomycin- $\mathrm{C}$ alone as adjuvant treatment for non-muscle-invasive bladder cancer (NMIBC). BJU Int. 107, 912-918 (2011).

77. Di Stasi, S. M. et al. Sequential BCG and electromotive mitomycin versus BCG alone for high-risk superficial bladder cancer: a randomised controlled trial. Lancet Oncol. 7, 43-51 (2006).

78. Oosterlinck, W. et al. Sequential intravesical chemoimmunotherapy with mitomycin $\mathrm{C}$ and bacillus Calmette-Guérin and with bacillus Calmette-Guérin alone in patients with carcinoma in situ of the urinary bladder: results of an EORTC genito-urinary group randomized phase 2 trial (30993). Eur. Urol. 59, 438-446 (2011).

79. Houghton, B. B. et al. Intravesical chemotherapy plus bacille Calmette-Guérin in non-muscle invasive bladder cancer: a systematic review with meta-analysis. BJU Int. 111, 977-983 (2013).

80. Bouffioux, C. et al. Adjuvant chemotherapy of recurrent superficial transitional cell carcinoma: results of a European Organization for Research on Treatment of Cancer randomized trial comparing intravesical instillation of thiotepa, doxorubicin and cisplatin. The European Organization for Research on Treatment of Cancer Genitourinary Group. J. Urol. 148, 297-301 (1992).

81. Bartoletti, R. et al. Intravesical gemcitabine therapy for superficial transitional cell carcinoma: results of a phase II prospective multicenter study. Urology 66, 726-731 (2005).

82. Addeo, R. et al. Randomized phase III trial on gemcitabine versus mytomicin in recurrent superficial bladder cancer: evaluation of efficacy and tolerance. J. Clin. Oncol. 28, 543-548 (2010).

83. Dalbagni, G. et al. Phase II trial of intravesical gemcitabine in bacille Calmette-Guérinrefractory transitional cell carcinoma of the bladder. J. Clin. Oncol. 24, 2729-2734 (2006).

84. Skinner, E. C. et al. SWOG S0353: Phase II trial of intravesical gemcitabine in patients with nonmuscle invasive bladder cancer and recurrence after 2 prior courses of intravesical bacillus Calmette-Guérin. J. Urol. 190, 1200-1204 (2013).

85. Barlow, L. J., McKiernan, J. M. \& Benson, M. C. Long-term survival outcomes with intravesical docetaxel for recurrent nonmuscle invasive bladder cancer after previous bacillus CalmetteGuérin therapy. J. Urol. 189, 834-839 (2013).

86. Sekine, H., Ohya, K., Kojima, S. I., Igarashi, K. \& Fukui, I. Equivalent efficacy of mitomycin $\mathrm{C}$ plus doxorubicin instillation to bacillus CalmetteGuérin therapy for carcinoma in situ of the bladder. Int. J. Urol. 8, 483-486 (2001).
87. Gontero, P. et al. The impact of intravesical gemcitabine and 1/3 dose Bacillus CalmetteGuérin instillation therapy on the quality of life in patients with nonmuscle invasive bladder cancer: results of a prospective, randomized, phase II trial. J. Urol. 190, 857-862 (2013).

88. McKiernan, J. M. et al. Phase I trial of intravesical docetaxel in the management of superficial bladder cancer refractory to standard intravesical therapy. J. Clin. Oncol. 24 , 3075-3080 (2006).

89. Lightfoot, A. J. et al. Multi-institutional analysis of sequential intravesical gemcitabine and mitomycin $\mathrm{C}$ chemotherapy for non-muscle invasive bladder cancer. Urol. Oncol. 32 , 35.e15-35.e19 (2014)

90. Steinberg, R. L. et al. Sequential intravesical gemcitabine and docetaxel for the salvage treatment of non-muscle invasive bladder cancer. Bladder Cancer (in press).

91. Gray, P. J. et al. Clinical-pathologic stage discrepancy in bladder cancer patients treated with radical cystectomy: results from the national cancer data base. Int. J. Radiat. Oncol. Biol. Phys. 88, 1048-1056 (2014).

92. Gray, P. J. et al. Use of potentially curative therapies for muscle-invasive bladder cancer in the United States: results from the National Cancer Data Base. Eur. Urol. 63, 823-829 (2013).

93. Quilty, P. M. \& Duncan, W. Treatment of superficial (T1) tumours of the bladder by radical radiotherapy. Br. J. Urol. 58, 147-152 (1986)

94. Gospodarowicz, M. K. et al. Bladder cancer: long term follow-up results of patients treated with radical radiation. Clin. Oncol. (R. Coll. Radiol.) 3 , 155-161 (1991)

95. Harland, S. J. et al. A randomized trial of radical radiotherapy for the management of pT1G3 NXMO transitional cell carcinoma of the bladder. J. Urol. 178, 807-813; discussion 813 (2007)

96. Weiss, C. et al. Radiochemotherapy after transurethral resection for high-risk T1 bladder cancer: an alternative to intravesical therapy or early cystectomy? J. Clin. Oncol. 24, 2318-2324 (2006).

97. Wo, J. Y. et al. The results of concurrent chemoradiotherapy for recurrence after treatment with bacillus Calmette-Guérin for non-muscle-invasive bladder cancer: is immediate cystectomy always necessary? BJU Int. 104, 179-183 (2009).

98. National Comprehensive Cancer Network ${ }^{\circledR}$. NCCN Clinical Practice Guidelines in Oncology (NCCN Guidelines ${ }^{\circledR}$ ) Bladder Cancer Version 1.2015 [online], http://www.nccn.org/ professionals/physician gls/pdf/bladder.pdf (2015).

99. Radiation Therapy Oncology Group. RTOG 0926 Protocol Information [online], http:// www.rtog.org/ClinicalTrials/ProtocolTable/ StudyDetails.aspx?study=0926 (2014).

100. Ping, S. Y., Wu, C. L. \& Yu, D. S. Sunitinib can enhance BCG mediated cytotoxicity to transitional cell carcinoma through apoptosis pathway. Urol. Oncol. 30, 652-659 (2012).

101. Chen, D. J. \& Huerta, S. Smac mimetics as new cancer therapeutics. Anticancer Drugs 20, 646-658 (2009)

102. Kemp, T. J. et al. Neutrophil stimulation with Mycobacterium bovis bacillus Calmette-Guérin (BCG) results in the release of functional soluble TRAIL/Apo-2L. Blood 106, 3474-3482 (2005)

103. Ludwig, A. T. et al. Tumor necrosis factor-related apoptosis-inducing ligand: a novel mechanism for Bacillus Calmette-Guérin-induced antitumor activity. Cancer Res. 64, 3386-3390 (2004). 
104. Jinesh, G. G., Chunduru, S. \& Kamat, A. M. Smac mimetic enables the anticancer action of BCG-stimulated neutrophils through TNF- $\alpha$ but not through TRAIL and FasL. J. Leukoc. Biol. 92, 233-244 (2012).

105. Mizutani, Y., Nio, Y., Fukumoto, M. \& Yoshida, O. Enhanced antitumor effect of Bacillus CalmetteGuérin in combination with fibrinogen on urinary bladder tumor. J. Urol. 151, 1420-1426 (1994).

106. Inman, B. A. et al. PD-L1 (B7-H1) expression by urothelial carcinoma of the bladder and BCG-induced granulomata: associations with localized stage progression. Cancer 109, 1499-1505 (2007).

107. US National Library of Medicine. ClinicalTrials. gov [online], https://clinicaltrials.gov/ct2/ show/NCT01560923 (2014).

108. Burke, J. M. et al. A first in human phase 1 study of CG0070, a GM-CSF expressing oncolytic adenovirus, for the treatment of nonmuscle invasive bladder cancer. J. Urol. 188 , 2391-2397 (2012).

109. Dinney, C. P. et al. Phase I trial of intravesical recombinant adenovirus mediated interferon$a 2 b$ formulated in Syn3 for Bacillus Calmette-Guérin failures in nonmuscle invasive bladder cancer. J. Urol. 190, 850-856 (2013)

110. Berger, A. P. et al. Photodynamic therapy with intravesical instillation of 5-aminolevulinic acid for patients with recurrent superficial bladder cancer: a single-center study. Urology 61 , 338-341 (2003).

111. Manyak, M. J. \& Ogan, K. Photodynamic therapy for refractory superficial bladder cancer: longterm clinical outcomes of single treatment using intravesical diffusion medium. J. Endourol. 17, 633-639 (2003).

112. Lee, J. Y. et al. Efficacy and safety of photodynamic therapy for recurrent, high grade nonmuscle invasive bladder cancer refractory or intolerant to bacille Calmette-Guérin immunotherapy. J. Urol. 190, 1192-1199 (2013).

113. Olsen, E. et al. Pivotal phase III trial of two dose levels of denileukin diftitox for the treatment of cutaneous T-cell lymphoma. J. Clin. Oncol. 19 , 376-388 (2001).

114. Kowalski, M. et al. A phase II study of oportuzumab monatox: an immunotoxin therapy for patients with noninvasive urothelial carcinoma in situ previously treated with bacillus Calmette-Guérin. J. Urol. 188, 1712-1718 (2012).

115. Chow, N. H., Chan, S. H., Tzai, T. S., Ho, C. L. \& Liu, H. S. Expression profiles of ErbB family receptors and prognosis in primary transitional cell carcinoma of the urinary bladder. Clin. Cancer Res. 7, 1957-1962 (2001).

116. Yang, X. et al. Diphtheria toxin-epidermal growth factor fusion protein DAB389EGF for the treatment of bladder cancer. Clin. Cancer Res. 19, 148-157 (2013).
117. Cho, S. K. et al. Functionalized gold nanorods for thermal ablation treatment of bladder cancer. J. Biomed. Nanotechnol. 10, 1267-1276 (2014).

118. Szlachcic, A. et al. FGF1-gold nanoparticle conjugates targeting FGFR efficiently decrease cell viability upon NIR irradiation. Int. J. Nanomedicine 7, 5915-5927 (2012).

119. Kawai, K., Miyazaki, J., Joraku, A., Nishiyama, H. \& Akaza, H. Bacillus Calmette-Guérin (BCG) immunotherapy for bladder cancer: current understanding and perspectives on engineered BCG vaccine. Cancer Sci. 104, 22-27 (2013).

120. Morales, A. et al. Efficacy and safety of MCNA in patients with nonmuscle invasive bladder cancer at high risk for recurrence and progression after failed treatment with bacillus Calmette-Guérin. J. Urol. http://dx.doi.org/10.1016/ j.juro.2014.09.109.

Author contributions

All authors contributed to researching the data for the article, discussing content and writing, as well as reviewing/editing the manuscript before submission.

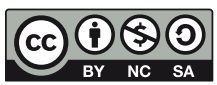

This work is licensed under a Creative Commons AttributionNonCommercial-Sharealike 4.0 Unported License. To view a copy of this license, visit https://creativecommons.org/licenses/ by-nc-sa/4.0/. 